\title{
Leve mejoría con la Adición de Memantine en pacientes con Alzheimer tratados con donepezilo.
}

\section{Objetivo:}

Comparar la eficacia y la seguridad del memantine en pacientes con enfermedad de alzheimer (EA) moderada a severa que reciben tratamiento con donepezilo.

Diseño:

Ensayo clínico, aleatorizado, doble ciego y controlado.

Lugar:

Estados Unidos, 37 centros, entre junio 2001 a junio 2002.

\section{Participantes:}

404 pacientes con EA moderada a severa, residentes en la comunidad, ambulantes, con cuidadores, en condiciones médicas estables, con Mini-Mental State Examination (MMSE) entre 5 y 14 , mayores de 50 años, en tratamiento con donepezilo por más de 6 meses con dosis estables durante los últimos 3 meses, sin cambios en las dosis de la medicación habitual. Los participantes no debían tener enfermedades activas, demencia complicada con otra enfermedad o Hachinski Ischemia Score mayor a 4.

\section{Intervención:}

Durante 24 semanas, al tratamiento habitual con donepezilo se agregó memantine, en dosis crecientes de 5 a 20 miligramos diarios en 203 pacientes y placebo en los restantes 201.

\section{Medición de resultados:}

Cambios en el SIB (Severe Impairment Battery) una medición para evaluar cognición en pacientes dementes severos y en el ADCSADL 19 (Modified 19 Item Alzeheimer Disease Cooperative StudyActivities of Daily Living Inventory) una medición de funcionalidad en estadios avanzados de demencia. Ambos a partir de una medición basal. El análisis fue por intención de tratar.

\section{Resultados principales:}

Hay un beneficio estadísticamente significativo en el grupo memantine utilizando la medición del SIB y el ADCS-ADL 19. Los efectos adversos fueron del $78 \%$ en el grupo memantine y $72 \%$ en el grupo placebo. El efecto adverso más frecuente fue la agitación en ambos grupos y la confusión se presentó en el grupo memantine con mayor frecuencia y significancia estadística.

\begin{tabular}{|c|c|c|c|}
\hline Score & $\begin{array}{l}\text { Memantine } \\
\text { [IC 95\%] }\end{array}$ & $\begin{array}{l}\text { Placebo } \\
\text { [IC 95\%] }\end{array}$ & $\mathbf{P}$ valor \\
\hline SIB cognición & $\begin{array}{l}\text { Basal } 78 \text { [75.8- } 80.2] \\
\text { Número pacientes } 198\end{array}$ & $\begin{array}{l}\text { Basal } 80[77.7-82.3] \\
\text { Número pacientes } 197\end{array}$ & \\
\hline $0=$ Mala Cognición & Cambio a las 24 Semanas & Cambio a las 24 Semanas & \\
\hline $100=$ Buena Cognición & $\begin{array}{c}1[(-0.4)-(2.4)] \\
\text { Número pacientes } 171\end{array}$ & $\begin{array}{c}-2.4[(-3.88)-(-0.92)] \\
\text { Número pacientes } 153\end{array}$ & $<.001$ \\
\hline $\begin{array}{c}\text { ADCS-ADL } 19 \\
\text { funcionalidad } \\
0=\text { Mala Funcionaliadad }\end{array}$ & $\begin{array}{l}\text { Basal } 35.5[34-37] \\
\text { Número de pacientes } 198\end{array}$ & $\begin{array}{l}\text { Basal } 35.8 \text { [ 34.3- } 37.3] \\
\text { Número de pacientes } 197\end{array}$ & \\
\hline 54=Buena Funcionalidad & $\begin{array}{c}\text { Cambio a las } 24 \text { Semanas } \\
-1.7[(-2.9)-(-0.7)] \\
\text { Número de pacientes } 172\end{array}$ & $\begin{array}{l}\text { Cambio a las } 24 \text { Semanas } \\
-3.3[(-4.4)-(-2.2)] \\
\text { Número de pacientes } 152\end{array}$ & .02 \\
\hline
\end{tabular}

\section{Conclusiones:}

Es el primer ensayo clínico publicado que examina la utilización de memantine en pacientes con EA que reciben dosis estables de donepezilo. Memantine fue mejor que el placebo para el tratamiento de la EA moderada a severa en pacientes de la comunidad mejorando la función cognitiva, las actividades de la vida diaria, la conducta y el estado clínico global.

\section{Comentario}

El tratamiento de la EA en los estadios moderados a severos se encuentra en estudio en los últimos tiempos. A la fecha, el donepezilo y el memantine por separado han demostrado una eficacia modesta para el tratamiento de la EA moderada-severa ${ }^{1,2 .} \mathrm{Al}$ actuar en diferentes sitios a nivel neuronal, se especuló si su asociación sería beneficiosa. Memantine es un antagonista no competitivo de los receptores N-metil-D-aspartato y bloquea los efectos de disfunción neuronal que pueden ocasionar niveles elevados de glutamato. Donepezilo es un inhibidor de la acetilcolinesterasa y aumenta la disponibilidad de moléculas de acetilcolina a nivel de los receptores postsinápticos. 2,3,4

El estudio comentado tiene un sesgo de selección* de la población, al no tener enfermedades activas concomitantes, ser pacientes ambulatorios y residentes de la comunidad. También al recibir dosis estables de donepezilo los participantes podrían experimentar mejor tolerancia y eficacia con el tratamiento por tener menos problemas médicos o menos deterioro del estado general en comparación con pacientes sin tratamiento previo por su EA.
El memantine podría tener efectos beneficiosos tanto como monoterapia o combinado con donepezilo en estadios avanzados de EA. La dosis inicial es de $5 \mathrm{mg}$ diarios con ascensos semanales de $5 \mathrm{mg}$ hasta llegar a $20 \mathrm{mg}$ diarios. El costo del tratamiento de donepezilo y memantine en forma conjunta es elevado, lo cual obliga en nuestro medio a realizar un análisis de costo/efectividad.

\section{Conclusiones del comentador}

Si bien el memantine podría agregar algún beneficio en pacientes con EA moderada a severa tratados con donepezilo, antes de indicar el tratamiento es importante establecer cuál es el significado clínico que tienen las pequeñas diferencias en las puntuaciones de las escalas en los pacientes y como impacta en la vida diaria del paciente y su entorno. También es relevante establecer la duración del tratamiento y como se evalúa la mejoría del paciente.

Dr. Javier Benchimol. [ Programa de Medicina Geriátrica. Servicio de Clínica Médica Hospital Italiano Buenos Aires.]

Benchimol J. Leve mejoría con la adición de Memantine en pacientes con Alzheimer tratados con donepezilo. Evid. actual. práct. ambul. $2004 ; 7: 106$. Comentado de: Tariot P, Farlow M, Grossberg G y col. Memantine treatment in patients with moderate to severe Alzheimer disease already receiving donepezil. JAMA 2004, Enero 21; 291: 317-324.

\section{Referencias}

1. Memantine in severe dementia: results of the M-BEST study (benefit and efficacy in severely demented patients during treatment with memantine). Winblad B, Poritis N. Int J Geriatr Psychiatry. 1999; 14: 135-146.

2. Memantine in moderate-to-severe Alzheimer's disease. Reisberg B, Doody R, R Stöfffler et al. N Engl J Med. 2003; 348: 1333-1341.

3. Pharmacological management of Alzheimer Disease: An uptodate. Hsiung G, Feldman H. Geriatrics and Aging, 2004; 7:247-250.

4. Medications for late-stage Alzheimer's disease: How should we use them? Lantz M. Clinical Geriatrics, 2004; 12: 34-37. 